\title{
Conducting Polymer Composites of Polypyrrole and Polyimide
}

\author{
F. Selampinar ${ }^{2}$, U. Akbulut ${ }^{3}$, L. Toppare \\ ${ }^{a}$ Department of Chemistry, Middle East Technical University, 06531 Ankara Turkey \\ ${ }^{b}$ Department of Chemistry, Bilkent University, 06533 Ankara Turkey
}

\begin{abstract}
A conducting composite of polypyrrole with a polyimide as the insulating matrix polymer was prepared via electrochemical methods. The characterization of the composite was done by FTIR, SEM and TGA studies. Conductivity and solubility studies together with spectroscopic methods reveal that a chemical interaction between the two polymers exists.
\end{abstract}

Key words: Electrochemical polymerization, insulating films, polypyrrole and derivatives.

\section{Introduction}

In the last decade, various electrically conductive polymers have been studied extensively. Among them a considerable number of works on polyheterocylics polymers such as polypyrrole, polythiophene and polyaniline were noticed because of their important advantages. These polymers are relatively stable under ambient conditions and can be conveniently synthesized via electropolymerization [1]. It has been accepted that the polypyrrole films have a number of potential technological applications in the areas of energy storage [2], solar energy conversion [3] and electrochromic display devices [4]. The mechanical properties can be enhanced by forming composite polymers from a conductive polymer and a conventional insulating polymer [5]. The most successful method to form the composite was found to be the electropolymerization of the pyrrole monomer on an electrode coated with an insulating polymer. As the insulating polymers polyvinyl chloride [5], polyvinyl alcohol [6], polyurethane [7] and polycarbonate [8] were commonly used.

Here we report the preparation of polypyrrole-polyimide (PPy/PI) composites via electrochemical synthesis. It is well known that polyimides have been the most studied polymeric materials for applications subjected to onditions of harsh environmental exposure. This type of m..terial has found applications as a high temperature adhesive in a number of aerospace structural parts and elcctronics/microelectronics because it has a continuous service temperature in the $250-350^{\circ} \mathrm{C}$ range and excellent electrical and mechanical properties. It was thought that these good properties may be combined with the electrical properties of polypyrrole. PPy/PI films have good electrical condustivity similar to that of pure PPy films and retain the favorable mechanical prof sities of the host PI films. The composite films were prepared by electrochemical oxidation of pyrrole on $\mathrm{Pl}$ coated electrode. The composite films were characterized by using SEM, FTIR and TGA techniques and compared with that of simple mechanical mixtures. The electroactivity of PI/PPy films was compared with that of PPy.

\section{Experimental}

PPy/PI composites were prepared by the electrochemical polymerization of pyrrole onto a PI precoated electrode at a constant potential of +1.3 volt versus $\mathrm{Ag}^{0} / \mathrm{Ag}^{+}\left(10^{-2} \mathrm{M}\right)$. The polyinide was dissolved in dinethylformanide (DMF) $(25$ m!g $\mathrm{L}^{-1}$ ) and used for dipcoating the Pt electrodes. The weight percent of polypyrrole in the composite was determined by weighing the electrodes before and after the polymerization. The structure of $\mathrm{PI}$ is given below:

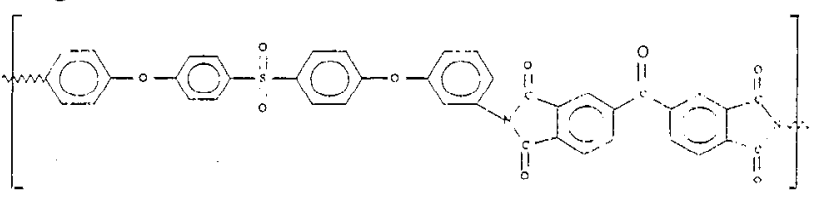

Polymerizations were carried out in a three-compartment cell equipped with $\mathrm{Pt}$ foils $\left(1.5 \mathrm{~cm}^{2}\right.$ each) as the working and counter electrodes and a capillary reference electrode $\left(\mathrm{Ag} / \mathrm{Ag}^{+}\right)$. The solvent-electrolyte couple was acetonitrile tetrabutylammonium tetrafluoroborate (TBAFB). Blank runs were also carried out in a medium where pyrrole was not present with PI coated electrode. The PI film is electrochemically stable and cannot bc electrooxidized during the electropolymerization of pyrrole. This is claimed by FT-IR of PI film before and after electrolysis. Also no weight loss was observed after at least one hour of electrolysis at the same conditions.

Electrical conductivities of samples were measured by four probe technique. The composites were characterized by FT-IR (Nicolet 510) and SEM(Cambridge S4-10). Thermal gravimetric analyses (TGA) were recorded on Du Pont 2100 instrument.

\section{Results and Discussion}

The electropolymerization of pyrrole on a PI coated electrode yielded a black film which can be easily peeled off from electrode. The conductivity of the PPy/PI films were given in Figure 1. The conductivity of the films depended on the content of PPy. Almost equal conductivities on both sides of the films 
suggest a homogeneity was achieved, at least, in terms of conductivity.

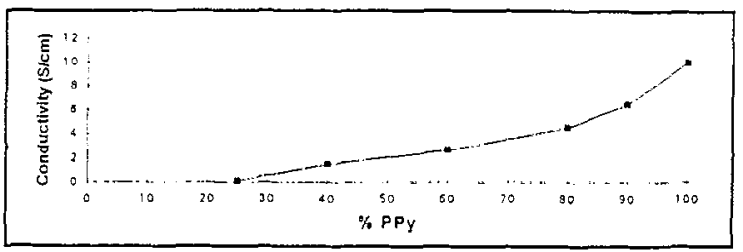

Fig. 1. Conductivity vs \% PPy

Cyclic voltammograms of solution containing $1 \times 10^{-3} \mathrm{M}$ Py and $0.1 \mathrm{M}$ TBAFB supporting electrolyte was performed with a potential scan from $-0.2 \mathrm{~V}$ to $+1.2 \mathrm{~V}$ versus $\mathrm{Ag} / \mathrm{Ag}^{+}$with a voltage scan rate of $1 \mathrm{~V} / \mathrm{sec}$. Figure 2 id shows the cyclic voltammetry of PPy at bare Pt. The plot reveals a peak for oxidation of the film at around $0.5 \mathrm{~V}$ on the anodic sweep and the corresponding reduction peak at around $+0.2 \mathrm{~V}$ in the cathodic sweep. In the case of PI coated electrode this electroactivity appears $+0.65 \mathrm{~V}$ oxidation and +0.2 reduction peaks. This small difference results from the fact that the pyrrole and the dopant ion have to diffuse through the insulating matrix to reach the $\mathrm{Pt}$ surface. Once this is accomplished the same type of electroactivity compared to bare Pt electrode was observed.

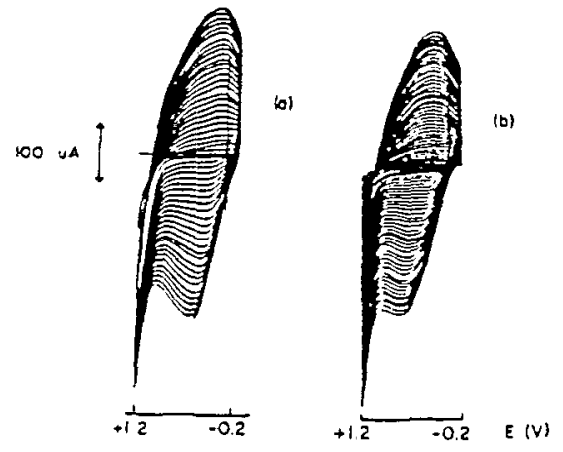

Fig, 2. Cyclic voltammogram of pyrrole in acetonitrile on a) bare $\mathrm{Pt}$ b) polyimide coated Pt electrodes.

When the films were washed with DMF for several weeks no marked changes were observed in terms of conductivity and weight.

The morphology of the composite films studied by scanning electron microscopy. The electrode side appears rather smooth with very little hollows. The smoothness indicates that conducting polymers starts to form active centers, from which the polymer diffuses through the host polymer spreading in all directions. On the solution side of the films many cauliflowerlike projections are observed. These projections are also observed in SEM of washed films. The similarity between washed and unwashed films suggests a single phase component.

In the FT-IR spectra of pure PPy, PI/PPy and washed PI/PPy film, the washed electrolytic film retains the peaks coming from Pl which is an indication that there is chemical interaction between PI/PPy. Both washed and unwashed films have 1780 $\mathrm{cm}^{-1}$ carbonyl asymetric, $1720 \mathrm{~cm}^{-1}$ carbonyl symmetric stretching and $1380 \mathrm{~cm}^{-1} \mathrm{C}-\mathrm{N}$ stretching. The strongest band $1080 \mathrm{~cm}^{-1}$ stands for $\mathrm{BF}_{4}$ dopant inn.

TGA curve of the composite was not simple addition of the two pure polymers which is shown by the mechanical mixture.
The mechanical mixture contains the same amount of PPy as the composite given in Figure 3. The PI and the mechanical mixture show two weight loss patterns the latter being around $570^{\circ} \mathrm{C}$ whereas the electrolytic film reveals a greater temperature stability as indicated by a sharp weight loss pattern at $670^{\circ} \mathrm{C}$.
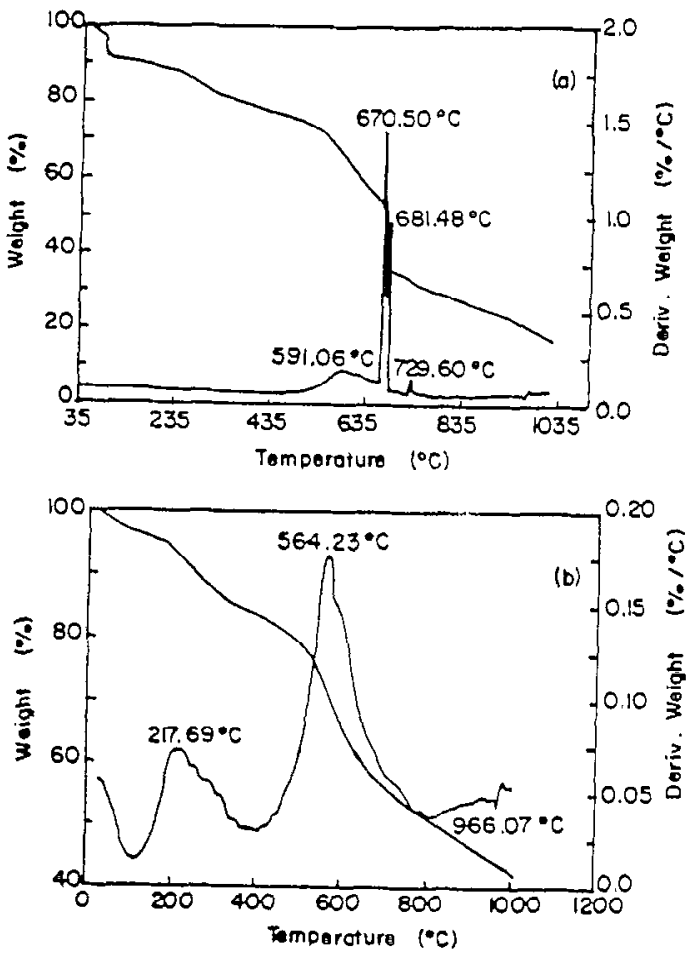

Fig. 3. Thermal Gravimetric Analysis a) PI/PPy electrolytic film b) mechanical mixture of PI and PPy.

\section{Conclusion}

Solubility tests together with thermal analyses bring enough evidence to believe that the PI/PPy composite film contains copolymers of PI and PPy to a certain extent.

\section{Acknowledgements}

We thank Dr. A. Güngör for the preparation of PI.

\section{References}

[1] A. F. Diaz and K. K. Kanazawa, J. Chem. Soc. Chem. Commun. (1979) 635.

[2] T. Osaka, K. Naoi, S. Ogano and S. Nakamura, $J$. Electrochem. Society, 134 (1987) 2096.

[3] T. Skotheim, O. Inganas, J. Prejza and I. Lundstrom, Mol. Cryst. Liq. Cryst. Str. 83 (1982) 329.

[4] A. F. Diaz, J. I. Castillo, J. A. Logan and W. Lee, J. Electroanal. Chem., 129 (1981) 115.

[5] M. DePaoli, S. Panero, P. Prosperi and B. Scrosati, Electrochim. Acta. 35 (1990) 1145.

[6] S. E. Lindsey, G. B. Street, Synth. Met. 10 (1984) 67.

[7] H. T. Chiu, J. S. Lin and C. M. Huang, J. Appl. Electrochem. 222 (1992) 358.

[8] H. L. Wang, L. Toppare and J. E. Fernandez, Macromolecules, 23 (1990) 1053 